Questions vives

\section{Questions Vives}

Recherches en éducation

$n^{\circ} 21 \mid 2014$

Le travail collectif des enseignants en question(s)

\title{
Champy-Remoussenard, P. (Éd.), En quête du travail caché : enjeux scientifiques, sociaux, pédagogiques
}

Toulouse : Octarès Éditions

Lucie Aussel

\section{(2) OpenEdition}

\section{Journals}

Édition électronique

URL : http://journals.openedition.org/questionsvives/1504

DOI : 10.4000/questionsvives. 1504

ISSN : $1775-433 \mathrm{X}$

\section{Éditeur}

Université Aix-Marseille (AMU)

Édition imprimée

Date de publication : 15 septembre 2014

ISBN : 978-2-912643-45-2

ISSN : $1635-4079$

Référence électronique

Lucie Aussel, « Champy-Remoussenard, P. (Éd.), En quête du travail caché : enjeux scientifiques, sociaux, pédagogiques », Questions Vives [En ligne], n 21 | 2014, mis en ligne le 15 septembre 2014, consulté le 22 septembre 2020. URL : http://journals.openedition.org/questionsvives/1504; DOI : https://doi.org/10.4000/questionsvives.1504

Ce document a été généré automatiquement le 22 septembre 2020.

\section{(†) $९$

Questions Vives est mis à disposition selon les termes de la licence Creative Commons Attribution Pas d'Utilisation Commerciale - Pas de Modification 4.0 International. 


\title{
Champy-Remoussenard, P. (Éd.), En quête du travail caché : enjeux scientifiques, sociaux, pédagogiques
}

Toulouse : Octarès Éditions

\author{
Lucie Aussel
}

\section{RÉFÉRENCE}

Champy-Remoussenard, P. (Éd) (2014). En quête du travail caché : enjeux scientifiques, sociaux, pédagogiques. Toulouse : Octarès Éditions.

1 Les auteurs de cet ouvrage collectif coordonné par Patricia Champy-Remoussenard s'intéressent aux enjeux des dimensions cachées du travail. Il fait suite à un ensemble de symposiums et de séminaires consacrés à cette thématique. Après la préface, dans les six chapitres qui structurent l'ouvrage : deux portent sur des milieux professionnels variés, trois s'appuient sur le travail des enseignants et des formateurs et un dernier s'intéresse aux enjeux d'une volonté de mise au jour des dimensions cachées du travail.

2 Yves Schwartz préface l'ouvrage. Il fait dans un premier temps une précision qui lui paraît importante: le travail n'est pas l'emploi. Bien que les contributions abordent principalement les dimensions cachées de ce cadre stricto sensu, il précise que l'emploi constitue une forme particulière de travail, celle la plus répandue dans nos sociétés "marchandes et de droit», mais qui ne saurait représenter la diversité existante. Il s'intéresse ensuite à l'appréhension de cette partie invisible du travail. Voir le travail en train de se faire suffit-il à accéder au travail caché ? Schwartz définit le travail comme « un moment de vie humaine » c'est-à-dire complexe, qui ne peut se restreindre à une succession de tâches (programme). Ainsi, il affirme qu'« un degré d'inapparence semble être une caractéristique "générique" de tout travail humain avec ses implications parfois douloureuse, voire dramatique». Cette affirmation repose, selon lui, sur la rencontre de trois types de professionnels (ergonomes, praticiens et 
médecins) qui permet de mettre en exergue l'importance à la fois des normes dans le travail (prescriptions, règles, procédures, etc.) et de leur accommodation pour que le travail soit vivable. Celle-ci à chaque fois nouvelle est appelée renormalisation, elle tient compte des choix faits par les travailleurs en fonction de leurs préférences, de leurs valeurs. Cette renormalisation est-elle visible ? L'auteur répond oui, à condition de la mettre en visibilité en tenant compte de deux écueils : il restera toujours une part d'invisible et le travail peut être favorisé ou bloqué par les travailleurs pour lesquels cette renormalisation est une marge de liberté ou inconsciente.

Dans le premier chapitre Pierre Imbert et Marc Durand proposent d'analyser le travail à partir de deux registres d'activités: utilitariste (manifeste) et anti-utilitariste (implicite) afin de comprendre la composante éducative dans le travail. Les auteurs se basent sur la triple obligation : donner, recevoir, rendre pour appréhender les relations au sein de l'activité étudiée. À partir de l'exemple de trois cas (en classe, dans un jury, parmi des accessoiristes) ils montrent comment l'anti-utilitarisme permet de comprendre et d'analyser le travail comme une totalité. Ils développent ensuite quatre apports. Le premier est méthodologique; la «clé de don» permet une analyse secondaire des matériaux de la recherche qui rend possible l'identification des composantes de l'activité humaine. La prise en compte des phénomènes implicites dans l'activité doit favoriser l'accès à l'aspect constructif (versus productif) du travail. Le deuxième apport est théorique, travailler l'anti-utilité implique «de considérer l'activité comme une totalité complexe et dynamique à différents niveaux d'organisation et à différentes échelles de temps ». Le troisième apport est ontologique, il concerne la compréhension de la relation éducative et invite à dépasser la conception symétrique des relations maitre/élève au sein de la classe au profit d'une conception alternativement dissymétrique entre donateurs (maître ou élèves) et donataires. Le dernier apport est éthique qui envisage des actions modestes, héroïques dans des moments anti-utilitaristes.

Dans le deuxième chapitre, Patricia Champy-Remoussenard aborde dans un premier temps le statut des dimensions dissimulées du travail. L'auteure, considérant le travail comme un fait social, distingue différentes façons de concevoir les zones d'ombres de cette activité (cachées, dévoilées, etc.). Elle propose dans la suite de sa contribution trois points de vue sur la nature de ces dimensions. Le premier aborde des pratiques cachées à partir de l'étude de l'activité de management qui participent, bien que contraire à des prescriptions officielles, à la reconnaissance du travail. Le deuxième point de vue traite des dimensions relationnelles souvent au cœur des activités " sociales ", mais qui demeurent cachées car peu valorisées. Le troisième revient sur la frontière floue entre des activités de la sphère privée et celles de la sphère du travail. Puis, elle définit les dimensions cachées du travail par leur nature générique (transférables), qu'elle distingue ensuite des dimensions clandestines (quasi impossible à officialiser). Enfin, elle énonce trois difficultés pour traiter ces dimensions : à la fois pour les faire formaliser, pour qu'elle soit prise en compte dans la formation professionnelle, mais aussi pour les communiquer comme résultats de recherche (danger quant aux résultats produit vis-à-vis des commanditaires de la recherche).

5 Françoise Lantheaume dans le troisième chapitre aborde l'invisible dans le travail des enseignants. Du point de vue de la sociologie de l'éducation, l'auteure met en avant différentes façons d'analyser l'invisibilité du travail (sélection des élèves, agir stratégique des acteurs, secret sur les façons de faire.) puis elle distingue dimensions 
cachées et invisibles. Les premières sont conscientes, non prescrites par l'institution ni mises en avant par les acteurs, mais pouvant néanmoins être décrites; les secondes sont inconscientes et non dicibles par les acteurs. Elle fait l'hypothèse que les prescriptions diverses des politiques éducatives entrent en tension avec la réalité du métier d'enseignant et les astreignent à développer « des pratiques invisibles à autrui ». Dans un premier temps, à travers trois exemples (enseignants de lycées professionnels, assistants pédagogiques et enseignants de l'éducation prioritaire) Lantheaume étudie l'influence de différentes formes de prescriptions: des sur prescription qui rendent flous les attendus engendrant de l'auto-prescription qui participe du développement des dimensions cachées du travail venant " combler les trous du normatif ». Dans un second temps, l'auteure met en avant l'injonction paradoxale de la production de résultats selon des normes standardisées (politique de grandeur) et la prise en compte de l'hétérogénéité des élèves (politique de proximité) favorisant l'incertitude des enseignants. Soumis également à des sur prescriptions ils développent des stratégies de gestion de cette incertitude par de l'auto-prescription et ainsi des dimensions cachées du travail.

6 Le quatrième chapitre concerne les ingéniosités clandestines qui permettent aux professionnels de tirer parti de l'incertitude. L'auteure, Nicole Mencacci, définit ces ingéniosités comme des savoirs habiles peu visibles car ils sont à la fois fugitifs, situés et appartenant au registre du "corps-soi ». Elle convoque le cadre théorique de la pensée Métis et de l'intelligence du Kaïros pour les caractériser. À travers une étude empirique basée sur des enregistrements vidéo, des observations et des entretiens post-repérage ancrage, l'auteure a pu mettre en évidence que la pensée Métis et l'intelligence Kaïros sont des habiletés fugitives incarnées par des enseignants sachant «ruser » c'est-à-dire saisir des trous de normes pour «jouer avec [leur] savoir et les attentes des apprenants». L'auteure pose ensuite trois questions : peut-on user de la ruse en éducation? Peut-on apprendre à être ingénieux ?... par l'observation, l'analyse de pratique? Elle montre dans un premier temps que la ruse peut être efficace en éducation à condition qu'elle soit perçue par les apprenants c'est-à-dire qu'il y ait un "accord intersubjectif pas nécessairement explicite». Elle avance alors que les différentes catégories de savoirs peuvent s'enseigner, mais pas la singularité de chaque praticien (signature professionnelle). De plus, elle évoque l'apprentissage par essaierreur car les enseignants sont seuls avec les élèves et doivent expérimenter par euxmêmes. Ainsi, elle conclut à une forme de formation hybride alliant observation et analyse de pratique.

7 Jean Clénet s'intéresse dans le cinquième chapitre aux désordres de la reconnaissance. Il questionne les dimensions cachées du travail de la pratique formative (autoréférences) et de la recherche associée. Il défend le statut flou/clandestin de certaines facettes $d u$ travail et propose une réflexion sur la posture éthique du chercheur face au sujet et à son autonomie. L'auteur se demande dans quelle mesure les dimensions cachées expliquent l'autonomie et le pouvoir d'agir des acteurs. Deux points sont ensuite développés, le premier aborde l'apprentissage par l'expérience, considérée comme une "connaissance empirique qui peut s'exprimer dans des conceptions renouvelées par un travail caché ». Le second soulève trois processus cachés par les sujets en formation : recherche de soi, résistance au social (organisation) et reconstruction. L'auteur questionne la méthodologie de recherche pour appréhender ce flou. Il s'appuie pour cela sur sa propre expérience de chercheur et avance une nécessaire prise en compte dans l'étude du travail en formation de processus « reliant 
sujet-objet-projet-situation-temps ». Puis, il met en avant l'importance du travail réflexif (flou dicible pouvant être rendu visible) qui redonne du pouvoir au sujet dans sa formation. Enfin, il s'intéresse à l'auto-organisation des sujets (activité intérieure et extérieure).

Le sixième et dernier chapitre est consacré à la part insue de l'activité. L'auteur, Richard Wittorki, précise la synonymie des termes «insu » et "invisible». Il choisit d'étudier les enjeux explicites et implicites de cette attention portée à l'invisible dans le travail. L'auteur définit la part insu du travail comme relevant de l'écart entre le prescrit et le réel : le "faire non prévu ». Le premier enjeu implicite de l'analyse de la part insue du travail, soulevé par l'auteur, est l'optimisation des formations (efficacité). Puis, trois nouveaux enjeux sont mis au jour. À l'initiative des groupes sociaux ou des individus, dans un premier temps, pour renforcer la légitimité de l'activité professionnelle. À l'initiative de l'organisation, dans un deuxième temps, afin de prendre la mesure d'une expertise professionnelle pour être capable de la transmettre aux nouveaux apprenants. À l'initiative des espaces de formation, dans un troisième temps, afin de susciter des apprentissages à partir de l'activité déployée. Enfin, R. Wittorki s'attache à explorer la question des outils méthodologiques et théoriques pour révéler la part invisible de l'activité. Il centre son propos sur les méthodes de coanalyse du travail (entretien d'auto-confrontation simple ou croisé, instruction au sosie, entretien d'explicitation, etc.) qui favorisent la compréhension de l'activité, mais aussi le développement professionnel du sujet. Enfin, il met en avant différents outils d'analyse existant à ce jour pour "lire» les dimensions cachées du travail (compétences incorporées, savoir caché dans l'agir, savoir pratique, d'expérience, etc.) La dernière partie de cet ouvrage est la postface, écrite par Pierre Pastré, intitulée : maitriser son activité ? L'auteur synthétise deux caractéristiques communes du travail caché portées par l'ensemble des contributions: il existe une part d'ombre dans l'activité de travail, elle peut être appréhendée que dans la diversité qu'elle représente (cachée, clandestine, implicite, tacite, incorporée, etc.) et il est important de l'illustrer avec des situations concrètes.

\section{AUTEURS}

\section{LUCIE AUSSEL}

Chercheure associée UMR EFTS - Université de Toulouse 